\title{
Evaluation of a genetic assay for canine transmissible venereal tumour
}

\section{diagnosis in Brazil}

Karina Ferreira de Castro*, Andrea Strakova, Mirela Tinucci Costa, Elizabeth P. Murchison

Corresponding author*: Karina Ferreira de Castro

Email: karfcastro@gmail.com

Address: Av. Belvedere, n. 1005/249 (Garden Village I). Postal code: 15057-400, São José do Rio Preto/São Paulo/Brazil.

This study was performed in Department of Veterinary Medicine, University of Cambridge, Madingley Road, Cambridge CB3 OES, UK

\section{Abstract}

The canine transmissible venereal tumour (CTVT) is a transmissible cancer that is spread between dogs by the allogeneic transfer of living cancer cells. The infectious agents in CTVT are the living cancer cells themselves, which are transmitted between dogs during coitus. CTVT first arose several thousand years ago and the disease has a global distribution and is frequently observed in dogs from Brazil. We evaluated the utility of a LINE-MYC quantitative PCR for diagnosis of CTVT cases in Brazil. Our analysis indicated that the LINE-MYC rearrangement was detectable in all CTVT samples but not in their corresponding hosts. This genetic assay proves to be a useful tool for providing a definitive molecular diagnosis of CTVT, which presents with varying degrees of aggressiveness and invasiveness in different host dogs and can therefore be a diagnostic challenge in some specific cases.

Keywords: Canine transmissible venereal tumour, transmissible cancer, oncology, molecular diagnosis, LINE-MYC. 


\section{Introduction}

The canine transmissible venereal tumour (CTVT) is a naturally occurring transmissible cancer that affects the external genitalia of both male and female dogs. The disease is spread by the allogeneic transfer of living cancer cells mainly during coitus but also through licking, biting and scratching. ${ }^{1,2,3}$ CTVT is the oldest known mammalian somatic cell lineage and genetic studies indicate that it originated more than 10,000 years ago., ${ }^{4,6}$ Rather than dying together with its original host, the cells of this specific cancer are still alive today through acquiring adaptations for cell transmission between hosts and for survival as an allogeneic graft. ${ }^{3}$ CTVT is frequently observed in dogs from Brazil, however, this disease has a global distribution and has been documented to be present in at least 90 countries on all six inhabited continents and its distribution is linked to the presence of free-roaming dogs. ${ }^{7}$ Important evidence for clonal transmission of CTVT was provided by the identification of a genomic rearrangement involving the insertion of a repetitive $1.5 \mathrm{~kb}$ DNA segment derived from a long interspersed nuclear element (LINE) upstream of the first exon of the c-myc gene. ${ }^{8}$ This genomic rearrangement has been identified in a large set of globally distributed CTVT tumors, but it has not been found in normal dogs, and it is considered to be diagnostic evidence for CTVT. ${ }^{4,5,9,10,11,12,13,14,15,16}$ It is possible that this rearrangement was present in the germline of the CTVT founder (i.e. the animal from whose somatic cells CTVT first emerged), that it occurred somatically during the development of the founding CTVT tumour, or that it occurred somatically in a CTVT clone that has subsequently achieved global distribution. ${ }^{17}$ The purpose of this study was to evaluate the utility of a LINE-MYC quantitative PCR (qPCR) for diagnosis of CTVT cases in Brazil. 


\section{Materials and Methods}

\section{Tumour and blood specimens}

Samples were obtained from 20 unrelated dogs with spontaneous CTVT diagnosed at the Veterinary Hospital "Dr Halim Atique" - Centro Universitário de Rio Preto (UNIRP), São José do Rio Preto, São Paulo, Brazil. The dogs were of different breeds, age and sex, as specified in Table 1. Tumour samples with an average size of $1 \mathrm{~cm}^{3}$ were collected by surgical excision from all 20 dogs and immediately stabilised by freezing in liquid nitrogen. These samples were collected before starting chemotherapy treatment. Two to five mililitres of peripheral blood were also collected from 17 of these dogs and mixed immediately with RNAlater solution in the proportion of 1:3 (Blood: RNAlater solution). This study was approved by the Animal Research Ethics Committee of the São Paulo State University at Jaboticabal, São Paulo, Brazil, under protocol number 24674/2012.

\section{Genomic DNA extraction}

DNeasy blood \& tissue kit $₫$ (Qiagen - product number 69506) was used to extract genomic DNA from both blood and tumour tissue according to the manufacturer's protocol. The DNA was quantified using a Nanodrop ND-1000 spectrophotometer and Qubit ${ }^{\circledR}$ assay (Life technologies - product number Q32851)

\section{Quantitative PCR}

The quantitative PCR (qPCR) was performed with two primer sets (see Table 2): one primer set was specific for B-ACTIN, and was used as a control for normalization; the second pair of primers spanned the LINE-MYC rearrangement junction, and their product is thus specific to CTVT. ${ }^{18}$ The qPCR was performed with SYBR $^{\circledR}$ Green Mix (Life Technologies - product number 4312704) with conditions described in Tables 3 and 4. qPCR was performed using an Applied Biosystems 
7900HT Fast Real-Time PCR system. Standard curves for LINE-MYC and B-ACTIN were generated using a CTVT sample, 29T. Relative LINE-MYC and B-ACTIN amplification values were estimated for each sample using the standard curve.

\section{Results}

\section{LINE-MYC rearrangement}

In order to confirm the CTVT status of all 20 samples, qPCR was performed for the LINE-MYC rearrangement. This procedure was conducted with CTVT and host samples. Results showed that the LINE-MYC rearrangement was detectable in all $20(20 / 20)$ CTVT samples but not in their corresponding hosts $(0 / 17)$ (Figure $1 \mathrm{~A}$ and 1B). The mean LINE-MYC:B-ACTIN ratio was 1.2527 in tumours and 0.0002 in hosts.

\section{Discussion/Conclusion}

CTVT represents the oldest known malignant cell in continuous propagation whereby a single malignant clone of cells has colonized dogs worldwide. Clinical history, signalment and cytological/histological features are often sufficient for diagnosis of CTVT. However, molecular biology can also be helpful in cases with an atypical presentation. The identification of a LINE element insertion near the MYC locus in the CTVT genome by PCR amplification is of diagnostic importance. The presence of detectable levels of LINE-MYC rearrangement observed in all CTVT samples and absence in their corresponding hosts confirm that this assay provided a diagnosis of CTVT. Our findings support published data indicating that the LINE-MYC rearrangement is conserved in CTVT and can be used clinically as a definitive diagnosis of CTVT. ${ }^{4,5,19}$ The variation in observed LINE-MYC:B-ACTIN DNA ratios between tumours identified in this study is probably due to to variable levels of normal host cells present in tumours; however, it is also possible that there is 
variation in LINE-MYC and/ or B-ACTIN copy number between tumours. It will be important to determine the limit of sensitivity of this assay to detect CTVT tumours with a high proportion of host cells. The mean ratio of LINE-MYC to B-ACTIN in CTVT tumours was 1.2527 , and probably reflects non-diploid copy number of LINEMYC and/or B-ACTIN in CTVT tumours, together with the presence of host cells. Molecular diagnosis of CTVT may be particularly useful in cases of CTVT with atypical presentation. For instance, CTVT with extragenital occurrence and generalised metastases, often become a diagnostic and clinical challenge, as it may be difficult to distinguish between CTVT and other canine round cell neoplasms. This genetic assay may, therefore, prove to be a very useful tool for providing a definitive diagnosis in these cases. In conclusion, as a specific molecular alteration in CTVT, the rearrangement of LINE-MYC was also identified in CTVT cells in dogs from Brazil and this genetic assay can be useful as definitive diagnosis of CTVT.

\section{References}

1. Cohen, D., 1985. The canine transmissible venereal tumour: a unique result of tumour progression. Advances in cancer research 43, 75-112.

2. Das, U., Das, A. K. (2000). Review of canine transmissible venereal sarcoma. Veterinary Research Communications 24, 545-556.

3. Strakova, A., Murchison, E. P. (2015). The cancer which survived: insights from the genome of an 11000 year-old cancer. Current Opinion in Genetics \& Development 30:49-55.

4. Murgia, C., Pritchard, J.K., Kim, S.Y., Fassati, A., and Weiss, R.A. (2006). Clonal origin and evolution of a transmissible cancer. Cell 126, 477-487.

5. Rebbeck, C.A., Thomas, R., Breen, M., Leroi, A.M., and Burt, A. (2009). Origins and evolution of a transmissible cancer. Evolution, 1-10.

6. Murchison, E.P., Wedge, D.C., Alexandrov, L.B., Fu, B., Martincorena, I., Ning, Z., Tubio, J.M., Werner, E.I., Allen, J., De Nardi, A.B., et al. (2014). Transmissible dog cancer genome reveals the origin and history of an ancient cell lineage. Science 343, 437-440. 
7. Strakova, A., Murchison, E. P. (2014). The changing global distribution and prevalence of canine transmissible venereal tumour. BMC Veterinary Research 10:168, 1-10.

8. Katzir, N., Rechavi, G., Cohen, J.B., Unger, T., Simoni, F., Segal, S., Cohen, D., and Givol, D. (1985). "Retroposon" insertion into the cellular oncogene cmyc in canine transmissible venereal tumor. Proceedings of the National Academy of Sciences of the United States of America 82, 1054-1058.

9. Katzir, N., Arman, E., Cohen, D., Givol, D., Rechavi, G. (1987). Common origin of transmissible venereal tumors (TVT) in dogs. Oncogene 1, 445-8.

10. Amariglio, E.N., Hakim, I., Brok-Simoni, F., Grossman, Z., Katzir, N., Harmelin, A., Ramot, B., and Rechavi, G. (1991). Identity of rearranged LINE/c-MYC junction sequences specific for the canine transmissible venereal tumor. Proceedings of the National Academy of Sciences of the United States of America 88, 8136-8139.

11. Choi $\mathrm{Y}$, Ishiguro N, Shinagawa M, Kim, CJ, Okamoto Y, Minami S, Ogihara k. Molecular structure of canine LINE-1 elements in canine transmissible venereal tumor. Anim Genet 1999; 30:51-3.

12. Chu RM, Lin CY, Liu CC, Yang SY, Hsiao YW, Hung SW, Pao HN, Liao KW. Proliferation characteristics of canine transmissible venereal tumor. Anticancer Res 2001; 21:4017-24.

13. Choi, Y., Kim, C.J. (2002). Sequence analysis of canine LINE-1 elements and p53 gene in canine transmissible venereal tumor. $J$ Vet Sci 3, 285-92.

14.Liao, K.W., Lin, Z.Y., Pao, H.N., Kam, S.Y., Wang, F.I., and Chu, R.M. (2003). Identification of canine transmissible venereal tumor cells using in situ polymerase chain reaction and the stable sequence of the long interspersed nuclear element. Journal of veterinary diagnostic investigation: official publication of the American Association of Veterinary Laboratory Diagnosticians, Inc 15, 399-406.

15.Park MS, Kim Y, Kang MS, Oh SY, Cho DY, Shin NS et al. (2006). Disseminated transmissible venereal tumor in a dog. J Vet Diagn Invest 18: 130-133.

16. Vázquez-Mota, N., Simón-Martínez, J., Córdova-Alarcon, E., Lagunes, L., Fajardo, R. (2008). The T963C mutation of TP53 gene does not participate in the clonal origin of canine TVT. Vet Res Commun 32, 187-191.

17. Murchison, E. P. (2009). Clonally transmissible cancers in dogs and Tasmanian devils. Oncogene 27, 19-30.

18. Rebbeck, C.A. (2007). Canine transmissible cancer: evolution of a selfish cell lineage (Unpublished doctoral thesis). Imperial College London, London, England.

19. Fonseca. L.S., Mota, L.S.L.S., Colodel, M.M., Ferreira, I., Brandão, C.V.S., Rocha, N.S. (2012). Spontaneous canine transmissible venereal tumor: 
association between different phenotypes and the insertion LINE-1/c-myc. Rev Colomb Cienc Pecu 25, 402-408.

Acknowledgements:

This work was supported by a Research Grant from the Royal Society (RG130615). 
Table 1. Summary of samples used in this study. Tumour tissue was collected from all 20 animals; matched blood was collected from 17 animals.

\begin{tabular}{ccccc} 
Sample & Breed & Sex & Age (years) & Tumour location \\
\hline 1 & Pit Bull & F & 2 & Vagina \\
2 & Mixed & F & 10 & Vagina \\
3 & Mixed & M & 4 & Penis \\
4 & Mixed & F & 5 & Vagina \\
5 & Mixed & F & 3 & Vagina \\
6 & Mixed & F & 5 & Vagina \\
7 & Mixed & M & 4 & Penis \\
8 & Mixed & F & 5 & Vagina \\
9 & Shih Tzu & F & 4 & Vagina \\
10 & Border Collie & F & 4 & Vulva/Vagina \\
11 & Shih Tzu & F & 7 & Vagina \\
12 & Mixed & M & 3 & Penis \\
13 & Mixed & F & 6 & Vagina \\
14 & Mixed & F & 4 & Vulva/Vagina \\
15 & Mixed & M & 3 & Penis \\
16 & Poodle & F & 4 & Vagina \\
17 & Mixed & M & 5 & Penis \\
18 & Mixed & F & 5 & Vagina \\
19 & Mixed & M & 4 & Nasal cavity \\
20 & Border Collie & M & 3 & Penis/Prepuce/Eyelid \\
\hline & & & &
\end{tabular}

Table 2. Primer sequences for $\mathrm{APCR}$ of LINE-MYC and B-ACTIN.

\begin{tabular}{lll}
\hline & \multicolumn{1}{c}{ Forward } & Reverse \\
\hline B- ACTIN & CTCCATCATGAAGTGTGACGTTG & CGATGATCTTGATCTTCATTGTGC \\
LINE-MYC & AGGGTTTCCCATCCTTTAACATT & AGATAAGAAGCTTTTGCACAGCAA \\
\hline
\end{tabular}


Table 3. qPCR reaction conditions.

\begin{tabular}{cc}
\hline qPCR Reagent & $\begin{array}{c}\text { Volume per } \\
\text { reaction }\end{array}$ \\
\hline SYBR Green Mix & $10 \mathrm{uL}$ \\
Primers (5uM/primer) & $2.4 \mathrm{uL}$ \\
gDNA (20ng/ul) & $0.5 \mathrm{uL}$ \\
Water & $7.1 \mathrm{uL}$ \\
\hline Total volume & $20 \mathrm{uL}$ \\
\hline
\end{tabular}

Table 4. qPCR amplification conditions*

\begin{tabular}{llc}
\hline \multicolumn{1}{c}{ Stage } & Temperature $\left({ }^{\circ} \mathrm{C}\right)$ & Time \\
\hline Initial denaturation & 95 & 10 minutes \\
40 cycles & 95 & 15 seconds \\
& 60 & 60 seconds \\
\hline
\end{tabular}

${ }^{\text {*}}$ Fluorescence was detected each cycle 
(A) LINE-MYC - Tumour

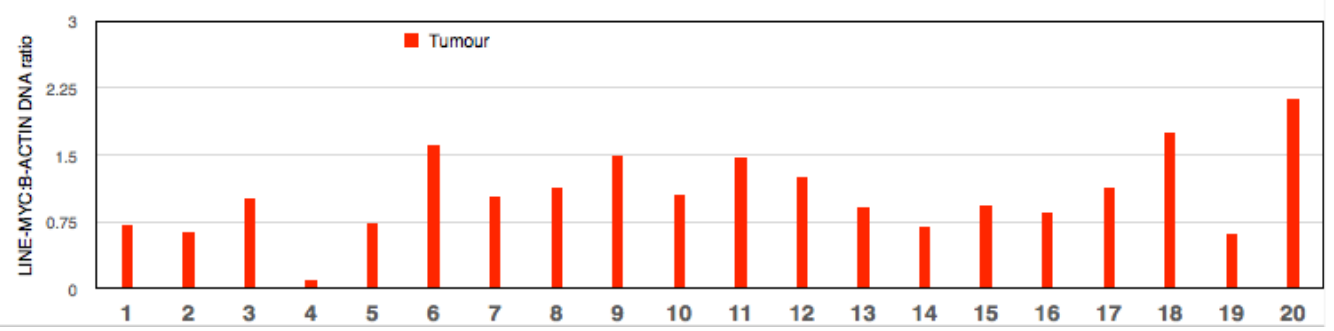

(B) LINE-MYC - Host

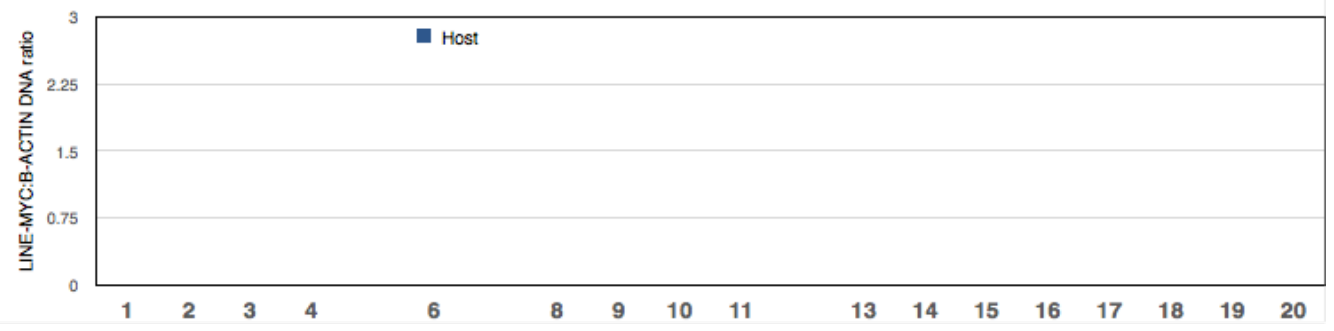

Figure 1. The bars represent LINE-MYC:B-ACTIN DNA ratio measured by qPCR in tumours (red) and hosts (blue). The LINE-MYC rearrangement was (A) detectable in all 20 CTVT samples and (B) absent in all hosts. Missing data from some hosts due to unavailability of samples are represented by absence of label $(5,7$ and 12$)$. 